\title{
A cystic fibrosis patient who is homozygous for the G85E mutation has very mild disease
}

\author{
Gillian Chalkley, Ann Harris
}

The most common mutation in the cystic fibrosis (CF) gene is the deletion of a phenylanaline residue at amino acid 508 of the protein $(\Delta \mathrm{F} 508) .{ }^{1}$ This lesion occurs in about $70 \%$ of northern European and North American CF chromosomes, though it is found at a much lower rate in southern Europe and the Middle East. ${ }^{23}$ The $\Delta \mathrm{F} 508$ mutation causes relatively severe disease generally with recurrent lung involvement and pancreatic insufficiency, as well as the raised sweat electrolytes that are diagnostic of CF. ${ }^{45}$ It is possible that certain other as yet undefined mutations may have significantly higher morbidity.

Correlations between genotype and phenotype for the majority of the mutations in the gene that have been defined by members of the CF Genetic Analysis Consortium have been slow to emerge. This is owing to the fact that most of the mutations have only been found in a very small number of independent CF chromosomes. Hence, the chances of defining a patient who is homozygous for one of these rare mutations is small, though genotype/phenotype correlations can be most clearly seen in these subjects. Compound heterozygotes, who carry different mutations on their two CF chromosomes, may present a much more complex clinical picture. For each pair of mutations in a subject it will ultimately be necessary to establish at the protein level, in the epithelia expressing the basic defect in vivo, the biological effects of different mutations.

A CF patient who has unusually mild disease has been identified as being homozygous for the

Division of Medical and Molecular Genetics, United Medical and Dental Schools of Guy's and St Thomas's Hospitals, 8th Floor Guy's Tower, London SE1 9RT

Correspondence to Dr Harris, Paediatric Molecular Genetics, Institute of Molecular Medicine, The John Radcliffe Hospital, Headington, Oxford OX3 9DU.

Received for publication 14 May 1991.

Accepted for publication 29 May 1991.
G386 $\rightarrow$ A mutation in exon 3 that results in a Gly $\rightarrow$ Glu change at amino acid 85 .

The proband is an 11 year old boy who was first diagnosed as having $\mathrm{CF}$ after presenting with a nasal polyp. He has sweat sodium values of $90 \mathrm{mmol} / \mathrm{l}$, mild lung disease, and is pancreatic sufficient. His parents are of Iranian origin and there is a family history of cousin marriages.

Based on predictions from the cDNA sequence of the cystic fibrosis transmembrane conductance regulator (CFTR), ${ }^{6}$ exon 3 of the CF gene would code for part of the amino-terminal cytoplasmic domain and part of the first loop of the first membrane spanning domain in the protein. While screening for mutations in exon 3 in a collection of non- $\Delta$ F508 CF chromosomes, in the South East Thames region, ${ }^{7}$ by amplification and mismatch detection, ${ }^{8}$ the proband was identified as carrying a mutation in exon 3 in at least one of his CF genes. Exon 3 was amplified by the polymerase chain reaction (PCR) using primers $3 \mathrm{i}-5$ and $3 \mathrm{i}-3$ (L-C Tsui, personal communication). PCR parameters were five minutes denaturation at $94^{\circ} \mathrm{C}$ followed by 30 cycles of $94^{\circ} \mathrm{C}$ denaturation for one minute, $60^{\circ} \mathrm{C}$ annealing for one minute, and $72^{\circ} \mathrm{C}$ extension for two minutes with a final elongation step of $72^{\circ} \mathrm{C}$ for five minutes. The $309 \mathrm{bp}$ amplified product was purified by Geneclean (Bio101) and subjected to chemical mismatch against exon 3 from a non-CF carrier. ${ }^{8}$

Fig 1 shows a mismatch reaction between exon 3 from a non-CF carrier control and eight CF patients including the proband (marked with an arrow). The exon 3 amplified product is seen at $309 \mathrm{bp}$ and the proband is seen to have an additional major band at about $105 \mathrm{bp}$. Direct sequencing of the amplified exon 3 from the proband and his parents was carried out ${ }^{9}$ using the $3 \mathrm{i}-5$ PCR primer as a template for the sequencing reaction. In fig 2 , top panel, the proband (cf) is seen to have a G386 $\rightarrow$ A substitution on both CF chromosomes when compared to control (c) DNA. In the lower panel, the parents of the proband ( $f=$ father, $m=$ mother) are both seen to be 


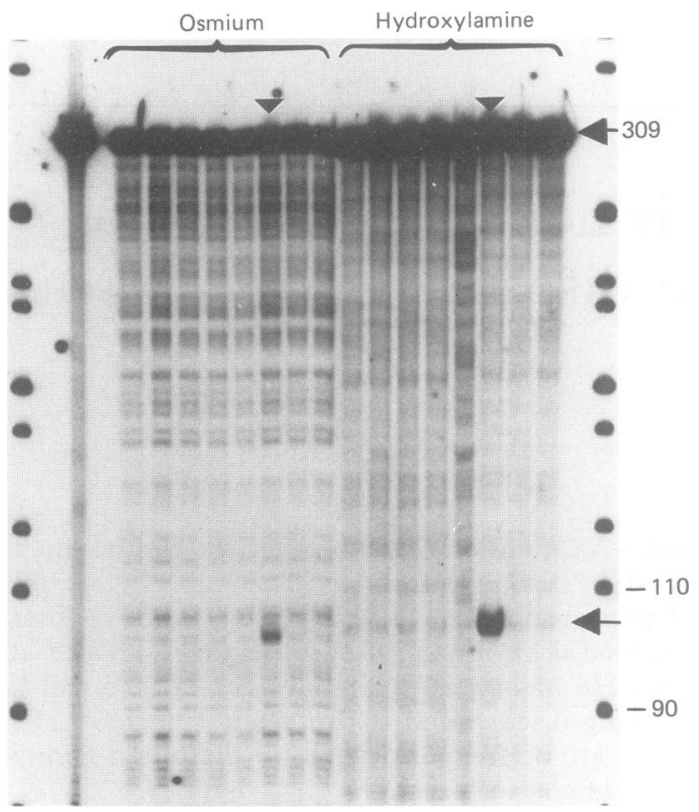

Figure 1 Autoradiograph of a $6 \%$ denaturing polyacrylamide gel showing a chemical mismatch reaction between control normal exon 3 and exon 3 from eight CF patients. Lane 1 shows the probe mismatched against itself, the next eight lanes show mismatches after osmium modification, and the last eight lanes after hydroxylamine modifications. The amplified DNA fragment containing exon 3 is 309 bp. Arrowheads denote the proband in lanes 7 and 15, where a mismatch band of about $105 \mathrm{bp}$ is seen.

heterozygous for $\mathrm{G} 386 \rightarrow \mathrm{A}$. The mutation is associated with a pXV2C/KM19 2/1 haplotype.

The G85E mutation was first defined by Zielenski et al. ${ }^{10}$ The substitution of glutamic acid for glycine at amino acid 85, which would result from the G386 $\rightarrow$ A mutation, is a relatively major change replacing a polar (uncharged) amino acid with a negatively charged one within the first membrane spanning domain of CFTR and might be expected to have a significant effect on the protein. However, the exceptionally mild disease manifested by the proband would suggest that this amino acid had a minor role in CFTR function. The only other CF patient with this mutation defined to date ${ }^{10}$ is of French Canadian origin. This subject has G386 $\rightarrow$ A in one $\mathrm{CF}$ gene and $621+1 \mathrm{G} \rightarrow \mathrm{T}^{10}$ in the other and has classical CF with pancreatic insufficiency (L-C Tsui, personal communication). It would be of interest to compare the severity of phenotype in this subject with CF patients who are homozygous for the $621+\mathrm{G} \rightarrow \mathrm{T}$ mutation, since the G85E mutation might be expected to exert a dominant effect in the compound heterozygote.
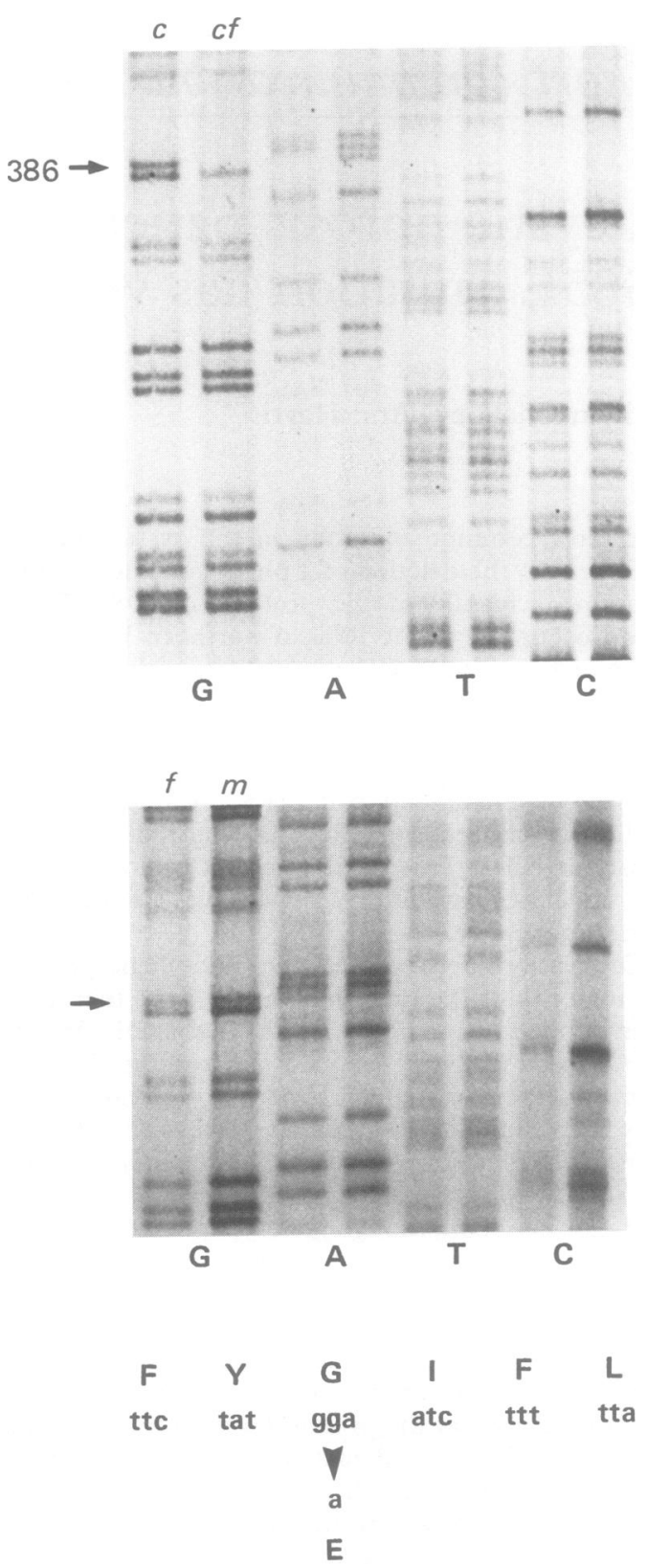

Figure 2 Sequencing of exon 3 from the 3i-5 primer. Top panel, control (c), proband ( $c f)$, arrow showing the G386 $\rightarrow$ A transition. Lower panel, parents of proband, $f=$ father, $m=$ mother. Below, nucleic acid and protein sequence around $G 386 \rightarrow A$ mutation.

The authors thank the Cystic Fibrosis Research Trust, the Generation Trust, and the Spastics Society for supporting this work, Frances Beards for help in defining non- $\Delta$ F508 patients, and $\mathrm{Dr} M$ Little for referral of patients. 
1 Kerem BS, Rommens JM, Buchanan JA, et al. Identification of the cystic fibrosis gene: genetic analysis. Science 1989; 245:1073-80.

2 Multiple authors. Worldwide survey of the delta 508 mutation. Report from the Cystic Fibrosis Genetic Analysis Consortium. Am f Hum Genet 1990;47:354-9.

3 Multiple authors. Gradient of distribution in Europe of the major CF mutation and of its haplotype. Hum Genet 1990;85:436-45.

4 Kerem E, Corey M, Kerem BS, et al. The relation between genotype and phenotype in cystic fibrosis: analysis of the most common mutation ( $\Delta \mathrm{F} 508$ ). N Engl f Med 1990;323:1517-22.

5 Borgo G, Mastella G, Gasparini P, Zorzanello A, Doro R, Pignatti PF. Pancreatic function and gene deletion $\triangle F 508$ in cystic fibrosis. I Med Genet 1990;27:663-9.

6 Riordan JR, Rommens JM, Kerem BS, et al. Identification of the cystic fibrosis gene: cloning and characterisation of complementary DNA. Science 1989;245:1066-73.

7 Harris A, Beards F, Mathew C. Mutation analysis at the cystic fibrosis locus in the British population. Hum Genet 1989;85:408-9.

8 Montandon AJ, Green PM, Giannelli F, Bentley DR. Direct detection of point mutations by mismatch analysis: application to haemophilia B. Nucleic Acids Res 1989;17:3347-58.

9 Green PM, Bentley DR, Misbashan RS, Nilsson IM, Giannelli F. Molecular pathology of haemophilia B. EMBO $f$ 1988;8:1067-72.

10 Zielenski J, Bozon D, Kerem B, Markiewicz D, Rommens JM. Tsui LC. Identification of mutations in exons 1 through 8 of the cystic fibrosis transmembrane conductance regulator (CFTR) gene. Genomics 1991;10:229-35. 